\title{
Helpa Tabelo por redukti Atmosferan Premon al la Marnivela Valoro.
}

De

W. Oishi.

Por redukti atmosferan premon al la marnivela valoro, oni uzas la konatan formulon

$$
h=18400(1+0.00367 \theta)\left(1+\frac{h}{6871104}\right) \log _{10} \frac{\mathrm{H}_{0}}{\mathrm{H}},
$$

kie oni nomas

H la atmosferan premon en la supra observejo kies altitudo estas h metroj,

$\mathrm{H}_{0}$ la atmosferan premon en la malsupra observejo je la marnivelo ( $\mathrm{H}$ kaj $\mathrm{H}_{4}$ estas valoroj korektitaj por temperaturo kaj graviteco),

$\theta$ la mezan centigradan temperaturon de la aertavoloj inter la du observejoj.

$$
\text { Nun } \begin{aligned}
\log _{10} \frac{\mathrm{H}_{0}}{\mathrm{H}} & =\frac{h}{18400(1+0.00367 \theta)\left(1+\frac{h}{6371104}\right)} \\
& =\frac{h}{18400+67.53 \theta+0.003 h} .
\end{aligned}
$$

Oni skribu

$$
\frac{h}{16400+67.53 \theta+0.003 h}=m .
$$

Oni do havas

$$
\log _{10} \frac{\mathrm{H}_{0}}{\mathrm{H}}=m \text {, aŭ } \frac{\mathrm{H}_{0}}{\mathrm{H}}=10^{m} .
$$


Sekve $\quad \mathrm{H}_{0}-\mathrm{H}=\mathrm{H}\left(10^{m}-1\right)$.

Kompreneble $\mathrm{H}_{0}-\mathrm{H}$ (tion oni nomu C) t.e. $\mathrm{H}\left(10^{m}-1\right)$ estas ja la reduktigo de la atmosfera premo al la marnivelo.

Plie estu $t$ kaj $t_{0}$ la aertemperaturoj en la supra kaj malsupra observejoj respektive. Se oni postulas ke

$$
t_{0}=t+\frac{1}{200} \cdot h \text { kaj } \theta=\frac{1}{2}\left(t+t_{0}\right),
$$

oni havas

$$
\theta=\frac{1}{2}\left\{t+\left(t+\frac{1}{200} h\right)\right\}=t+0.0025 h .
$$

Metinte anstataŭ $\theta$ en (2) g̀ gian valoron de (5), oni havas

$$
m=\frac{h}{18400+67.53 t+0.1718 h} .
$$

Uzante (6) ni kalkulas la valorojn de $10^{m}-1$ por diversaj altitudoj kaj diversaj gradoj de temperaturo. La rezultaton prezentas la tabelo III. La saman tabelon por la altitudoj de $0-200^{m} \mathrm{mi}$ jam antaŭ multaj jaroj publikigis en tiu ĉi jurnalo (22a jaro, no 5 ). Per la tabelo III oni povas facile kalkuli la valoroin de $\mathrm{C}=\mathrm{H}\left(10^{m}-1\right)$ por iu ajn altitudo malgranda ol $500^{\mathrm{m}}$.

Ekzemple estu $h=268.5$. Per interpolacio el la tabelo III, ni povas trovi la valorojn de $10^{m}-1$ respondantajn al $\mathrm{h}=268.5$, kiujn ni donas en la tabelo $\mathrm{I}$.

Tabelo I.

\begin{tabular}{rrrrrr}
$t$ & $10^{m}-1$ & $t$ & $10^{m}-1$ & $t$ & \multicolumn{1}{c}{$10^{m}-1$} \\
$-20^{\circ}$ & 0.036826 & $0^{2}$ & 0.034084 & $25^{\circ}$ & 0.031183 \\
-15 & 36100 & 5 & 33462 & 30 & 30660 \\
-10 & 35402 & 10 & 32861 & 35 & 30155 \\
-5 & 34730 & 15 & 32281 & 40 & 29666 \\
& & 20 & 31722 & &
\end{tabular}

Plie uzante la tabelon I oni kalkulas la valorojn de 


\section{$-3-$}

$\mathrm{C}=\mathrm{H}\left(10^{m}-1\right)$ por diversaj valoroj de atmosfera premo. La tabelo II estas la rezultato, per kiu oni povas tre facile trovi la reduktigon al la marnivelo por iu ajn valoro de la temperaturo kaj la atmosfera premo.

\begin{tabular}{|c|c|c|c|c|c|c|c|c|c|}
\hline & & & & $=$ & & & & & 8. \\
\hline $\mathrm{H}$ & 680 & 690 & 700 & 710 & 720 & 730 & & 750 & \\
\hline 20 & 25.04 & $25.4 \mathrm{I}$ & 25.78 & 26.15 & 26.5 & 6.88 & 7.25 & 7.62 & \\
\hline 15 & 24.55 & $24.9 \mathrm{r}$ & 25.27 & 25.63 & 25.99 & 26.35 & $26.7 \mathrm{r}$ & 27.08 & \\
\hline & $24 . \mathrm{C}$ & $24 \cdot 43$ & 24.78 & 25.14 & 25.49 & 25.84 & 26.20 & 26.55 & \\
\hline & 23.62 & $23.9^{6}$ & $24.3 \mathrm{r}$ & 24.66 & 25. & 25.35 & 25.70 & 26 & \\
\hline 0 & 23.18 & 2 & 23.86 & 24.20 & 24. & 24.88 & 25.22 & 25 & \\
\hline 5 & 22 & & 23. & 23.76 & & 24 & 24.76 & & \\
\hline 10 & 22.35 & 22.67 & 23.00 & 23.33 & 23. & 23. & $24.3^{2}$ & 24.65 & \\
\hline 15 & & & & & & & & 24.21 & \\
\hline 20 & 21.5 & 21.89 & $2 \mathrm{I}$ & 2 & 22.8 & 23.16 & 23.47 & 23. & \\
\hline & & & in & 22. & & & & & \\
\hline & 20.85 & & $2 \pi .46$ & 7 & .08 & $22.3^{3}$ & 22.69 & 3.6 & \\
\hline & & & & & & & & 306 & \\
\hline & & & & & & & .05 & .25 & \\
\hline
\end{tabular}

Se oni volus havi la tabelon II rekte el (6), oni nepre bezonus almenaŭ tri horojn por la kalkulado, sed nur duonhoron per la helpo de la tabelo III. Krom tio, oni bezonas en la unua okazo la scion de logaritmo, kontraŭe en la dua nur simplaj aritmetikaj operacioj sufiças.

Tokyo, Decembro I9I 7 . 
Tabelo III (A). $\quad\left(10^{\mathrm{m}}-1\right) \times 10^{6}$.

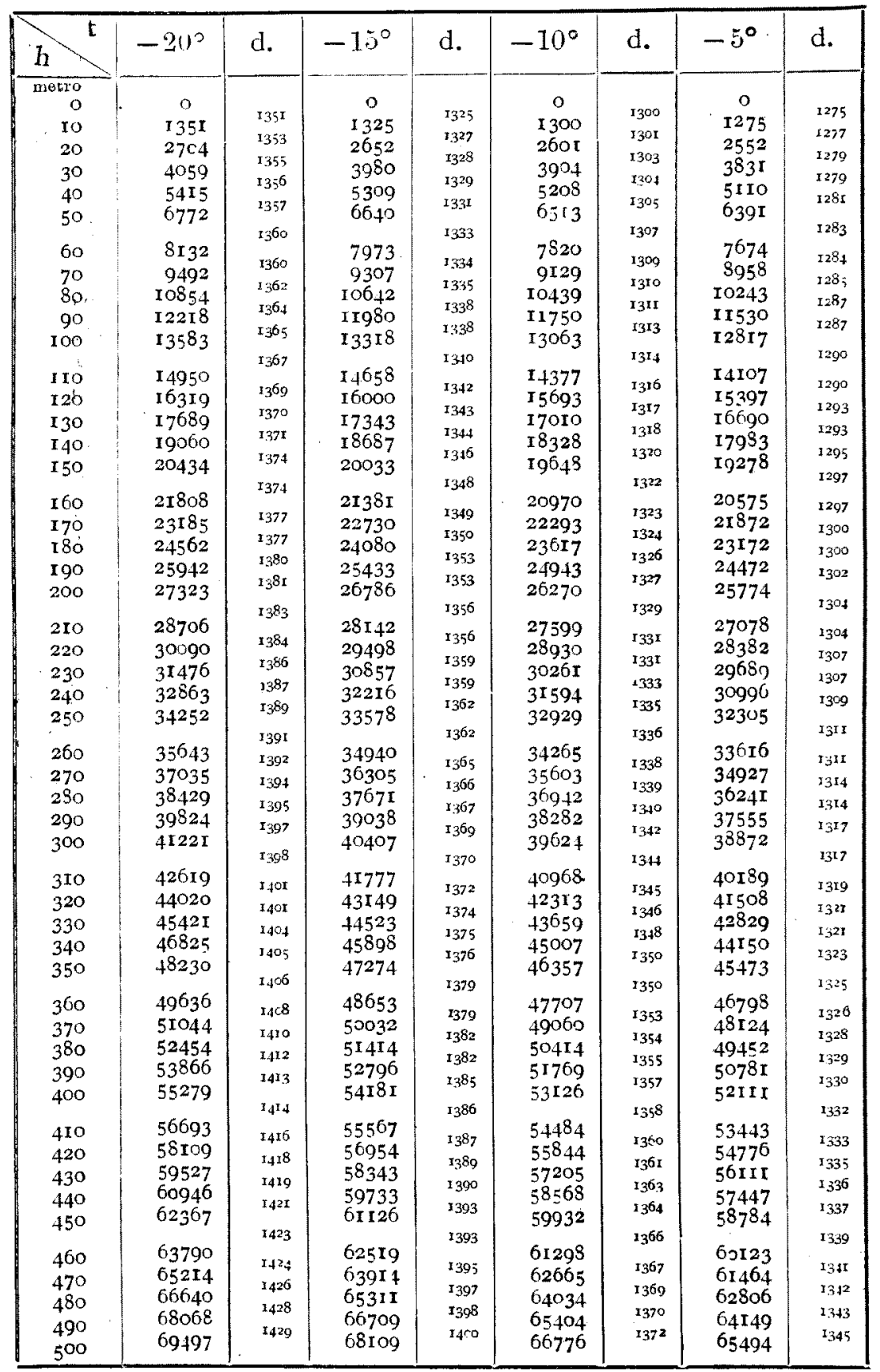


Tabelo III (B). $\quad\left(10^{\mathrm{m}}-1\right) \times 10^{6}$.

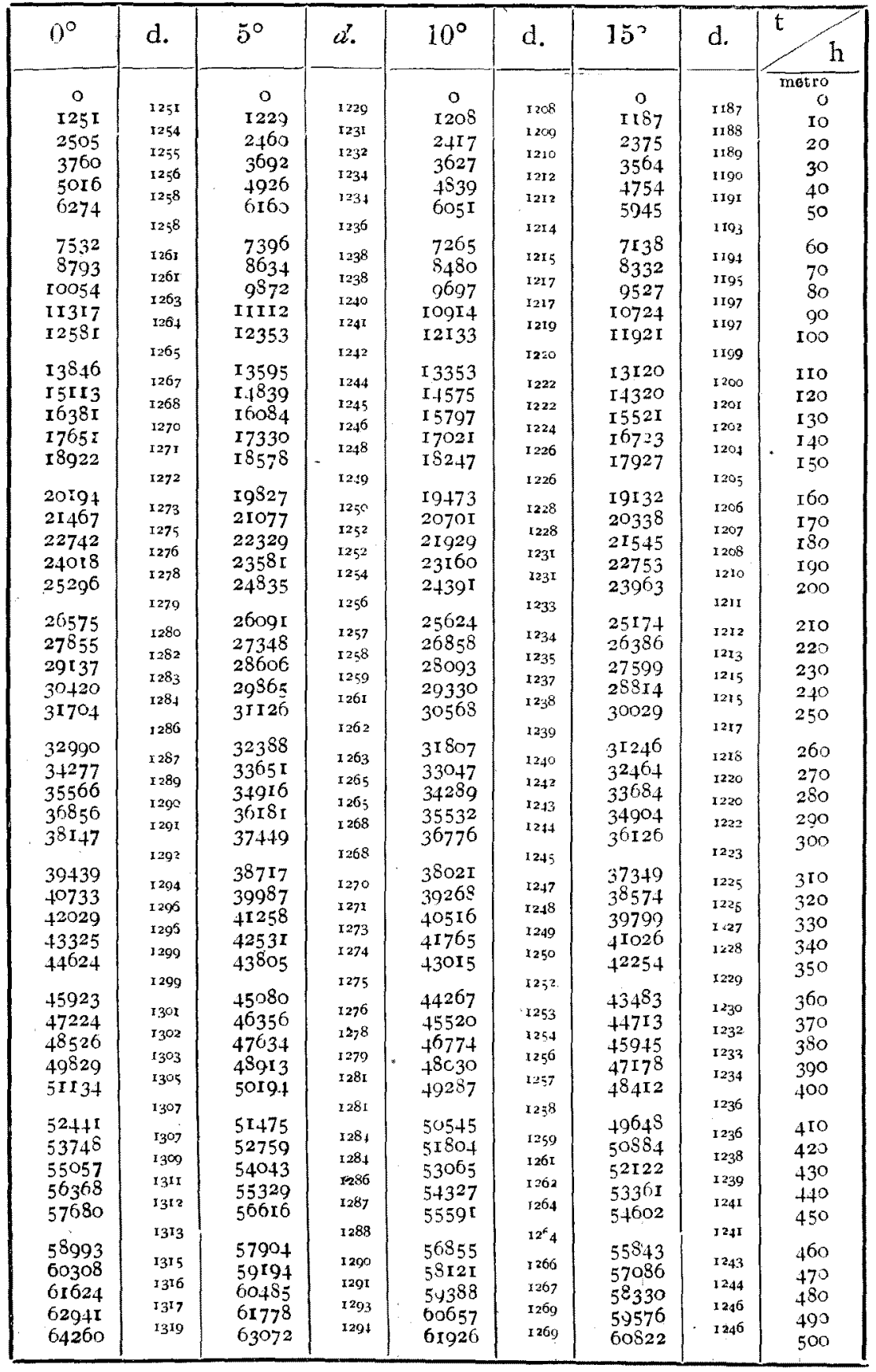


Tabelo III (C). $\quad\left(10^{\mathrm{m}}-1\right) \times 10^{6}$.

\begin{tabular}{|c|c|c|c|c|c|c|c|c|c|c|}
\hline$h^{t}$ & $20^{\circ}$ & d. & $25^{\circ}$ & d. & $30^{\circ}$ & d. & $35^{\circ}$ & d. & $40^{\circ}$ & d. \\
\hline metro & & & & & & & & & & \\
\hline $\begin{array}{r}0 \\
10\end{array}$ & $\begin{array}{c}0 \\
\text { I } 666\end{array}$ & 1166 & $\begin{array}{c}0 \\
17\end{array}$ & 1147 & $\begin{array}{c}0 \\
128\end{array}$ & 1228 & $\begin{array}{c}0 \\
\text { I }\end{array}$ & 1109 & $\begin{array}{c}0 \\
0\end{array}$ & 1092 \\
\hline 20 & 2334 & II 8 & $\begin{array}{l}11+7 \\
2295\end{array}$ & 1148 & $\begin{array}{l}128 \\
2257\end{array}$ & 1129 & $\begin{array}{l}1109 \\
2220\end{array}$ & IIII & $\begin{array}{l}1092 \\
2185\end{array}$ & $\mathrm{r} 693$ \\
\hline 30 & 3503 & 1169 & $\begin{array}{l}2295 \\
3+14\end{array}$ & 1149 & 3387 & $1+30$ & 3332 & IIIZ & $\begin{array}{l}2105 \\
3278\end{array}$ & rog3 \\
\hline 40 & 4673 & $\mathrm{rr}_{7} \mathrm{O}$ & 4594 & II 50 & 4518 & $x^{2} \mathbf{3} \mathrm{I}$ & $\$ 444$ & 1412 & 4373 & rogs \\
\hline 50 & 5844 & II 71 & $57+5$ & $115 x$ & 5650 & 1132 & 5558 & 314 & 5469 & $\operatorname{Icg} 5$ \\
\hline 60 & 7016 & 1172 & 6808 & 1153 & 6783 & 1133 & 6673 & III5 & 6566 & 1097 \\
\hline 70 & $8 \mathrm{IS9}$ & 1173 & $805 \mathrm{I}$ & I153 & 7918 & II35 & 7788 & II 15 & 7663 & 1007 \\
\hline So & 9364 & $\begin{aligned} 1175 \\
\end{aligned}$ & 9206 & 1155 & 9053 & 1135 & 8905 & III7 & 8762 & 3099 \\
\hline 90 & 10540 & $\begin{array}{l}1576 \\
1176\end{array}$ & 10362 & $115^{6}$ & IOI90 & 1137 & 10023 & $\begin{array}{l}1108 \\
1519\end{array}$ & 9862 & \pm 100 \\
\hline 100 & $\operatorname{II} 716$ & $\begin{array}{l}1170 \\
1178\end{array}$ & II 5 I 8 & 1156 & 11327 & 1137 & I I 12 & & rog63 & IIOI \\
\hline IIO & I 2894 & & $\mathrm{I} 2676$ & 1150 & 12466 & $1_{30}$ & 12262 & 1525 & I 2065 & Inoz \\
\hline 120 & 14073 & 1179 & $\mathrm{I}_{3} 8_{3} \mathrm{f}$ & 1160 & I 36006 & $I_{10}^{\circ}$ & $133^{8} 3$ & $\begin{array}{r}1121 \\
+122\end{array}$ & I 3168 & $\mathrm{IrC3}_{3}$ \\
\hline I30 & 15254 & 188 & 14996 & $\begin{array}{l}1100 \\
I \times 6 t\end{array}$ & $I+746$ & $\begin{array}{l}\mathrm{II} \neq 0 \\
x+y^{2}\end{array}$ & I +505 & $\begin{array}{l}1122 \\
1123\end{array}$ & $\mathrm{r}_{4272}$ & $\begin{array}{l}\mathrm{IIO}_{4} \\
\mathrm{y}\end{array}$ \\
\hline I & $\begin{array}{l}\mathrm{I} 6+35 \\
\end{array}$ & 1183 & I 6157 & I 63 & I 5888 & ז1 6 & I 5628 & 1524 & I 5377 & II 106 \\
\hline I 50 & 17618 & 1184 & I7320 & $\begin{array}{r}163 \\
5163\end{array}$ & $1703 \mathrm{I}$ & III & 16752 & $r 126$ & 16483 & IIOF \\
\hline 160 & 188.12 & 1185 & $18+83$ & $1 \times 65$ & $\operatorname{IS} 176$ & $\mathrm{rL}_{45}$ & I 7878 & 1126 & I 7590 & Iros \\
\hline I 70 & 19987 & I186 & I9643 & 1166 & I932I & І1 & r $900_{4}$ & 1127 & 18698 & $\begin{array}{l}1109 \\
I 109\end{array}$ \\
\hline 180 & 21173 & ris 88 & 20814 & 1167 & 20467 & $x_{4} 8$ & 20131 & 1129 & I9807 & IIIO \\
\hline I9o & 22361 & I 88 & 2 I98I & 1169 & $\begin{array}{l}21615 \\
22763\end{array}$ & 1148 & $\begin{array}{l}21260 \\
22300\end{array}$ & $11_{30}$ & 20917 & IIII \\
\hline 200 & 23549 & 1190 & $23 \times 50$ & 1169 & $22 / 03$ & 1150 & 22390 & $x \times 30$ & & IIII \\
\hline 210 & 24739 & IIGI & 24319 & $w=0$ & 23913 & $5 x=$ & 23520 & $11_{32}$ & 23140 & $t+t 3$ \\
\hline 220 & 25930 & II 92 & 25489 & 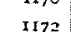 & 63 & 10 & 24652 & ${ }_{11_{3} 2}$ & 24253 & $\begin{array}{l}1113 \\
x I I 4\end{array}$ \\
\hline 230 & 27122 & 1193 & $2666 \mathrm{I}$ & $\begin{array}{r}177 \\
1573\end{array}$ & 26215 & $\begin{array}{l}1153 \\
1153\end{array}$ & $257^{84}$ & ${ }_{11} 4$ & 25367 & $\begin{array}{l}3 x+6 \\
3 x 6\end{array}$ \\
\hline $2+0$ & $283 \div 5$ & 1591 & 27834 & 1173 & 27368 & 1154 & 26918 & $\times 1 \times 35$ & 26483 & IIT6 \\
\hline 250 & 29509 & 1195 & 29007 & $x+76$ & 28522 & $11_{55}$ & 28053 & $113^{6}$ & 27599 & 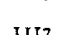 \\
\hline 260 & 30705 & 1197 & $3018_{3}$ & 1176 & 29677 & $\times 157$ & 29139 & 1137 & 28716 & 1117 \\
\hline $27^{\circ}$ & 31902 & 1198 & $3 \times 359$ & I17? & 30834 & $\begin{array}{l}1157 \\
1157\end{array}$ & 30326 & $\begin{array}{r}37 \\
1538\end{array}$ & 29834 & It1 8 \\
\hline 280 & 33100 & 1199 & 32536 & 1178 & $3199 \mathrm{I}$ & 1158 & 3146.1 & $I x_{39}$ & 30954 & 1120 \\
\hline 290 & 34299 & 1201 & $337 \mathrm{r}_{4}$ & 1180 & $33 \times 49$ & $\times 60$ & 32603 & $x x_{4}$ & 32074 & $\begin{array}{l}1120 \\
1122\end{array}$ \\
\hline 300 & 35500 & 1201 & 34894 & $118 \mathrm{i}$ & 34309 & $116 \mathrm{~T}$ & 33743 & $I r_{4} I^{\prime}$ & 33 I96 & 1122 \\
\hline 350 & $3670 \mathrm{I}$ & 1203 & 36075 & 1182 & 35470 & $x+65$ & $3488+$ & ${ }_{1142}^{2}$ & 34318 & 1122 \\
\hline $3^{20}$ & 37904 & 1204 & 37257 & 1183 & $3603 \mathrm{I}$ & $\begin{array}{r}1 \times 6 \\
\times 53\end{array}$ & 36026 & IftA & 3544 & $1 \times 24$ \\
\hline 330 & 39108 & $\mathrm{I} 2$ & $3^{3} 440$ & 1184 & 37794 & $\begin{array}{l}\text { II } 64 \\
\end{array}$ & 37170 & Is & 365 & 1124 \\
\hline 340 & 40313 & I206 & 39624 & $118 ;$ & 38958 & 1565 & 38314 & 1145 & 3769 & 1126 \\
\hline 350 & $4^{1} 519$ & 72 & 40809 & IIS & 40123 & 1167 & 39460 &  & $388 \mathrm{I}$ & 1125 \\
\hline 360 & 42726 & 1209 & 41996 & & 41290 & & 40607 & & 390 & 1128 \\
\hline 370 & & 5210 & $43 \times 83$ & 118 & & $\begin{array}{l}107 \\
\text { r } 68\end{array}$ & 41754 & & 41075 & 1129 \\
\hline 380 & & I2II & 44 & Ispo & 43625 & $\$ 170$ & 42903 & & 42204 & I 129 \\
\hline 390 & & 1212 & 455 & $\times 19 \mathrm{I}$ & 44795 & $x, 70$ & 44053 & 1150 & 43335 & I 137 \\
\hline 400 & 47568 & $12 \times 4$ & 46753 & IIg? & 45965 & 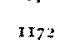 & 45204 & & 44467 & $x_{332}$ \\
\hline 410 & 48782 & $I_{2} \mathrm{I}_{4}$ & 47945 & III94 & 47 & & 46356 & & 45600 & $1 x_{33}$ \\
\hline 420 & 49996 & & 49139 & II & 48 & $\begin{array}{r}173 \\
1174\end{array}$ & & II53 & 46734 & 1134 \\
\hline 430 & $5 \times 2 \times 2$ & $\begin{array}{l}1210 \\
1217\end{array}$ & 50333 & III & & $\mathrm{II}_{5}$ & & 1154 & +7869 & 1135 \\
\hline 440 & $5^{2}$ & I219 & 5I 529 & 1197 & & 1176 & $498+8$ & $x+57$ & 49005 & ${ }_{11}^{16}$ \\
\hline $45^{\circ}$ & 53648 & & 52726 & I 198 & 51835 & 408 & 50975 & & $501+2$ & 5137 \\
\hline 460 & 54867 & & 53924 & & 53013 & & 52132 & $\pi=8$ & 51280 & $113^{8}$ \\
\hline 470 & 56088 & $122 \mathrm{I}$ & $55 x$ & 199 & $5+191$ & I 178 & 53290 & $\mathrm{II} 58$ & 52419 & 1159 \\
\hline 480 & 57309 & 1221 & 56323 & 12 & $5537 \mathrm{I}$ & 站 & 544 & troo & 53559 & $11_{40}$ \\
\hline 490 & $5^{3} 53^{2}$ & 1223 & 57525 & 2002 & 56552 & 1r8. & 556 & II & $5470 \mathrm{I}$ & $I^{I_{42}}$ \\
\hline 500 & 59757 & 1225 & 58728 & & 57733 & & 56772 & & $55^{8}+3$ & $\pi \times 42$ \\
\hline
\end{tabular}

